\title{
Aging behavior of porous silicon electrochemically etched with the aid of $\mathbf{Z n}$
}

\author{
K. Y. Suh, Y. S. Kim, and Hong H. Lee ${ }^{\text {a) }}$ \\ School of Chemical Engineering, Seoul National University, Seoul 151-742, Korea
}

(Received 7 June 2001; accepted for publication 16 July 2001)

\begin{abstract}
Aging behavior of red, green, and blue photoluminescence from porous silicon formed by electrochemical etching aided with zinc has been studied over a 3 month time span. Time-resolved photoluminescence spectra and decay dynamics have revealed that there are two radiating states in $\mathrm{Zn}$-aided porous silicon. One is the quantum confinement and the other is the oxygen-related defect. For green emission, the wavelength shifts from 520 to $420 \mathrm{~nm}$ and its photoluminescence spectra and decay behavior become very similar to those of blue emission. (C) 2001 American Institute of Physics. [DOI: 10.1063/1.1402976]
\end{abstract}

\section{INTRODUCTION}

Since strong visible luminescence from porous silicon (PS) has been reported, various luminescent recombination models based on quantum size effects, ${ }^{1}$ siloxene derivatives, ${ }^{2}$ and oxygen-related defects ${ }^{3}$ have been proposed. To clarify the photoluminescence (PL) mechanism, extensive studies have been carried out for the aging ${ }^{4,5}$ and transient decay behavior of PS. ${ }^{6-10}$ Typical aging experiments of redemitting PS have shown that the peak shows no blueshift and the intensity decreases gradually with aging. ${ }^{4}$ However, the aging behavior of green and blue emission, in particular that of green, has received little attention due to lack of proper preparation methods. ${ }^{5}$ For the transient decay dynamics of red emission, several authors reported multiexponential time decays with faster decay rates monitored at higher energies. ${ }^{6-8}$ These results have been suggested as a support for the quantum confinement model where smaller particles are predicted to have faster decay rates. However, Gole et $a .^{2}$ noted that the typical multiexponential time decays are also expected of any localized radiative center or defect state, including the fluorphors associated with the siloxene derivatives.

It is well known that the lifetime changes from microseconds for red emission located around $2.0 \mathrm{eV}^{6-8}$ to picoseconds to nanoseconds for blue emission located around $2.7-3.0 \mathrm{eV} .^{9-10}$ On the other hand, these kinds of materials can have a monoexponential PL decay with a microsecond lifetime for the blue PL according to the study of the PL of amorphous $\mathrm{SiO}_{2}$ and $\mathrm{SiO}_{x} .{ }^{11}$ Although there is no consensus about the origin of blue PL, it is assumed that the short lifetime originates from the oxygen-related defect. ${ }^{3}$

Recently, we reported ambient full-color photoluminescence from porous silicon through electrochemical etching aided by an "oxidative" metal such as $\mathrm{Zn}$ without any postanodizing treatment. ${ }^{12}$ The emission can be tuned to any wavelength in the visible range by simply changing the anodizing current density from $20 \mathrm{~mA} / \mathrm{cm}^{2}$ (red) to 100

\footnotetext{
a) Author to whom correspondence should be addressed; electronic mail: honghlee@plaza.snu.ac.kr
}

$\mathrm{mA} / \mathrm{cm}^{2}$ (blue). The origin of the ambient full-color PL and experimental procedure can be found elsewhere. ${ }^{12}$

In this article, we report the aging behavior of red, green, and blue PL from $\mathrm{Zn}$-aided porous silicon observed over a time span of 3 months.

\section{RESULTS AND DISCUSSION}

Figure 1 shows the aging behavior of the red, green, and blue photoluminescence over a period of 91 days. For the red emission, the peak shifted from 623 to $582 \mathrm{~nm}$ after 58 days and no further shifting occurred thereafter. Unfortunately, the as-prepared green photoluminescence is not stable in the ambient environment such that the green peak slowly shifts to blue and gets pinned at around $420 \mathrm{~nm}$. On the other hand, only a slight change in the peak wavelength and the peak width occurred in the case of the blue emission.

The Gaussian peak analysis reveals that each spectrum of the aged sample consists of two Gaussian peaks except for the red. The half widths and the shapes of the two peaks are found to remain the same throughout the aging for the green and blue-emitting PS, which is an indication that there may be two emission mechanisms. The change of the peak wavelength with time is shown in Fig. 2. The red emission consists of only one Gaussian peak and its peak location changes from 623 to $582 \mathrm{~nm}$ with almost no change after 58 days. For the green, the main peak is located at $525 \mathrm{~nm}$ and the minor is at $440 \mathrm{~nm}$ initially. The initial main peak becomes the minor peak with aging and the wavelength changes to 475 $\mathrm{nm}$, as shown in Figs. 1 and 2. The initial minor peak at 440 $\mathrm{nm}$, on the other hand, becomes the major peak after aging and the wavelength reduces to $420 \mathrm{~nm}$. For the blue, the initial major and minor peaks remain the same even after aging. The major peak wavelength simply changes from 423 to $415 \mathrm{~nm}$ with aging and the corresponding change in the minor peak is from 489 to $450 \mathrm{~nm}$. In all cases, further change with time is insignificant after 58 days.

In order to elaborate on the emission mechanism, PL spectra of as-prepared PS samples for $t_{d}=0$ and $t_{g}=100 \mathrm{~ns}$ are shown in Fig. 3. Here, $t_{d}$ is the delay time, which is defined as the time delay between the excitation laser pulse 

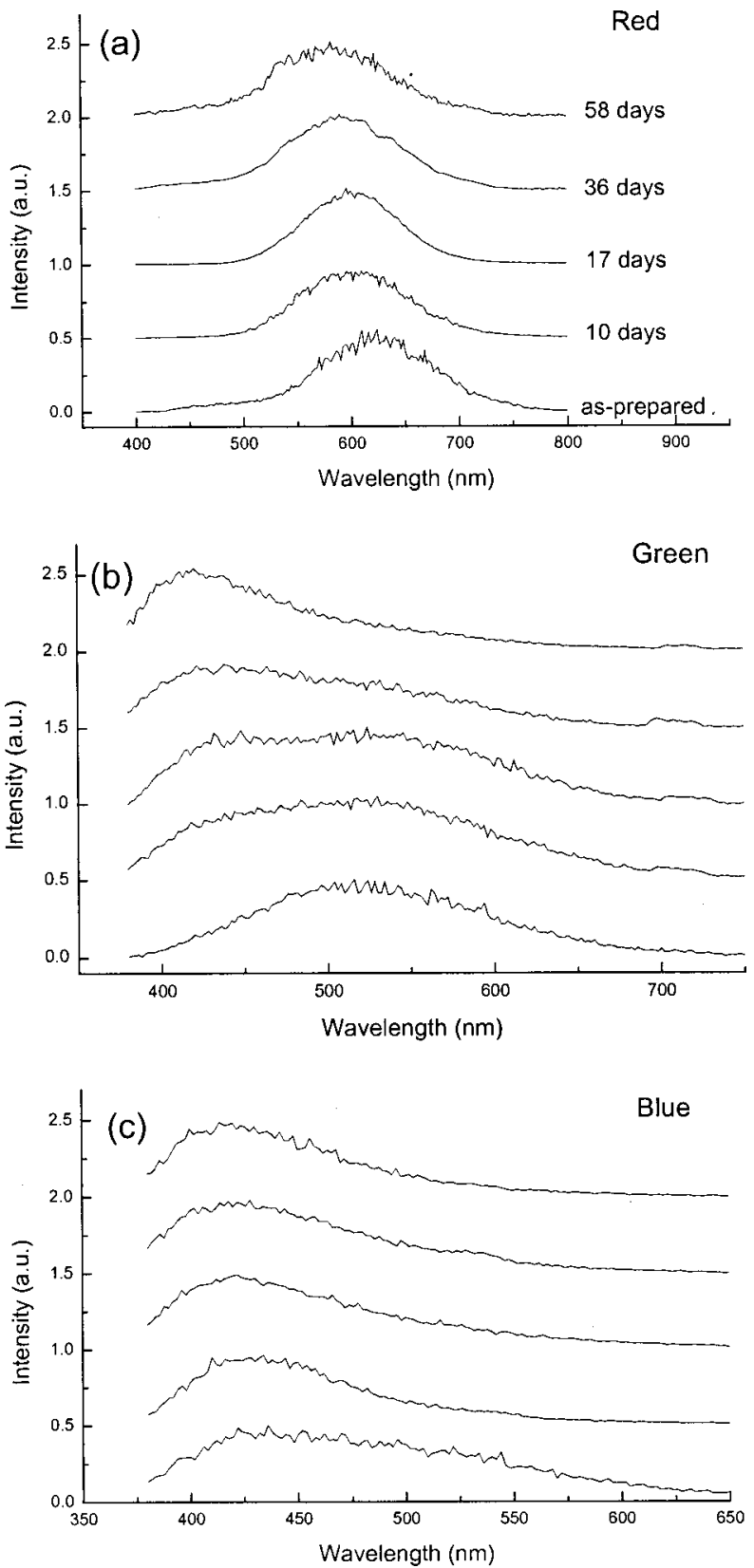

FIG. 1. PL spectra of aged Zn-aided PS samples of: (a) red, (b) green, and (c) blue as a function of aging time. The spectra are offset for comparison.

and a gate pulse and $t_{g}$ is the time interval of detection. For each figure, time-resolved PL (TRPL) spectra are also shown for $t_{d}=200 \mathrm{~ns}$ and $t_{g}=1 \mu \mathrm{s}$, where the spectrum for blue emission is magnified ten times. For all TRPL spectra, the intensities are very weak by 1 order of magnitude compared with that for $t_{d}=0$ and $t_{g}=100 \mathrm{~ns}$ and PL peaks are detected at longer wavelengths. For red emission, the spectrum for long-time delay consists of three Gaussian peaks, which are located at $539 \mathrm{~nm}(9.5 \%), 588 \mathrm{~nm}(44.7 \%)$, and $667 \mathrm{~nm}$ $(45.8 \%)$, respectively. For green emission, the spectrum consists of two Gaussian peaks, which are located at $443 \mathrm{~nm}$ $(6.9 \%)$ and $584 \mathrm{~nm}(93.1 \%)$, respectively. For blue emission, the spectrum also consists of two Gaussian peaks, which are located at $432 \mathrm{~nm}(59.8 \%)$ and $573 \mathrm{~nm}(40.2 \%)$, respectively. These results reveal that there are many radiating

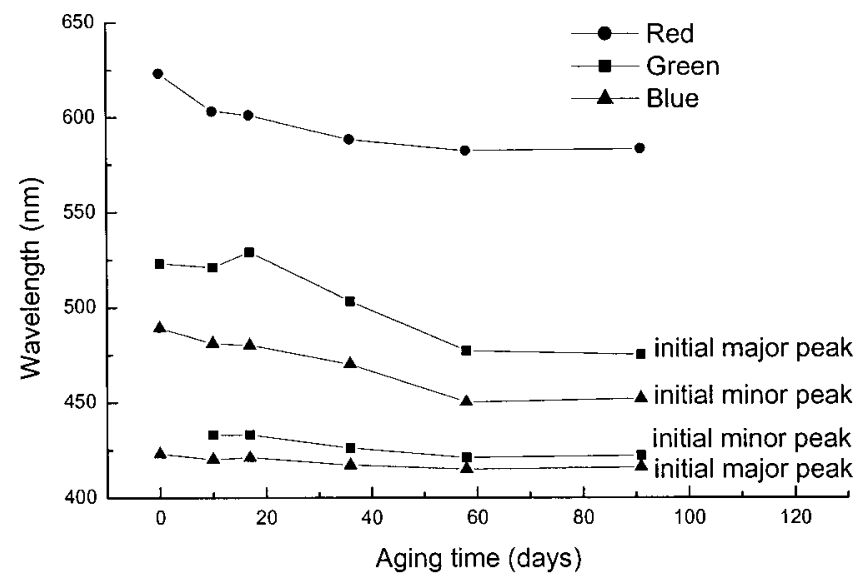

FIG. 2. Aging behavior of peak energy of Zn-aided PS samples. For the green and blue emission, the peak is deconvoluted into two Gaussian peaks.

states in freshly etched Zn-aided porous silicon, especially for the red emission.

As judged from the aging behavior and TRPL, one mechanism is operative for the red emission, whereas two mechanisms are for the green and blue emissions, although there is initially only one mechanism operative for the green. The dominance of one mechanism over the other in terms of emission intensity changes for the green but not for the blue emission.

The low etching current density applied to form the redemitting PS leads to a low porosity with the result that the microstructure is rather flat compared with that resulting with a higher current density. Such a relatively compact microstructure is apparently resistant to further oxidation once a thick oxygen-passivated layer forms. As a result, the peak wavelength blueshifts a little in the initial period of aging but then remains unchanged thereafter. The so-called, selflimiting oxidation process ${ }^{13}$ may be responsible for the behavior. The process is one in which oxidation can only progress up to a point where the stress generated by oxidation creates a strain barrier that would not allow any further oxidation.

For the green, a higher current density applied leads to a higher porosity and a much more open microstructure. This higher porosity brings with it finer nanoparticles in the size range corresponding to the green emission. As was the case in the red emission, the nanoparticles undergo oxidation by air and the corresponding blueshift occurs with aging. Once a highly strained oxide layer forms, the peak wavelength changes little with time. Apparently, oxygen-related defects are generated simultaneously as the oxidation of the Si nanoparticles takes place. ${ }^{14}$ The wavelength and half width of this new blue peak are nearly the same as those of the initially blue-emitting PS.

One remarkable observation regarding the aging behavior is the pinning of the wavelength of any blue emission at around $420 \mathrm{~nm}$, whether the emission is initially blue or green. This pinning is a strong indication that the light emission is associated with the oxygen-related defects since quantum confinement cannot cause the pinning at the specific wavelength of $420 \mathrm{~nm}$. In support of this conclusion, we 

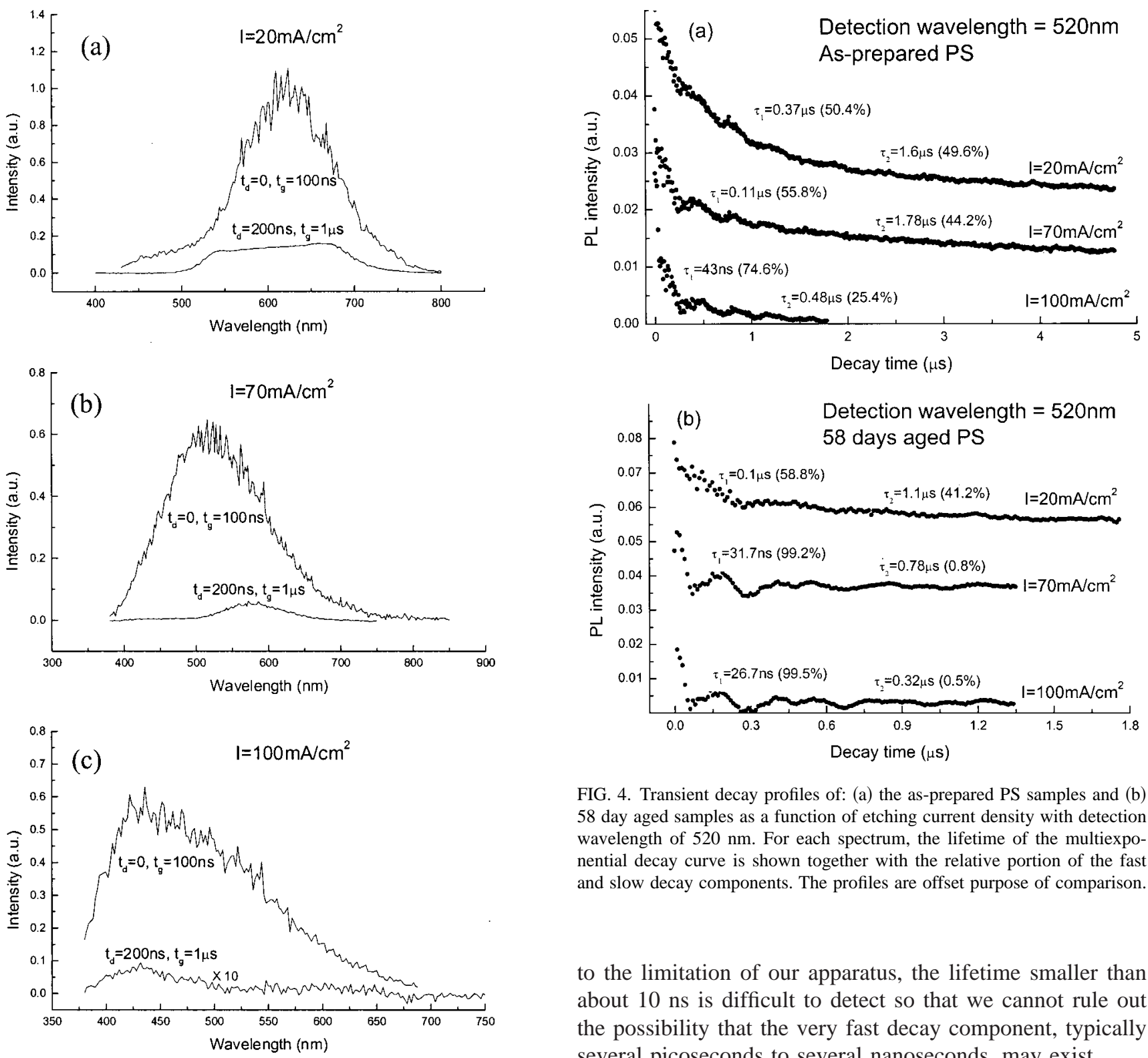

FIG. 3. Ambient room temperature TRPL spectra of the as-prepared PS samples formed by $\mathrm{Zn}$-aided electrochemical etching. The delay time and gate width are shown.

have recently found that the peak and shape of the PL spectra from blue-emitting PS almost coincides with that of the PL from defective oxide films prepared by plasma treatment. ${ }^{15}$

Figure 4(a) shows typical examples of PL decay profiles at a detection wavelength of $520 \mathrm{~nm}$. In our PS samples, the lifetime increases as the detection wavelength increases, which agrees with earlier reports. ${ }^{6,7}$ Every PL decay profile was nonexponential so that it was fitted to the following two exponential functions (curve fitting): $I=A_{1} \exp \left(-t / \tau_{1}\right)$ $+A_{2} \exp \left(-t / \tau_{2}\right)$. Here $I$ is the PL intensity, $A_{1}$ and $A_{2}$ are the prefactors, $\tau_{1}$ and $\tau_{2}$ are the lifetimes, and $t$ is the decay time. For each profile, the profile consists of a fast and a slow decay component and the portion of the fast component increases as the etching current density increases. For blue emission, the major lifetime is $43 \mathrm{~ns}$, which is a little larger than the value reported earlier. ${ }^{9,10}$ It is noted here that owing

FIG. 4. Transient decay profiles of: (a) the as-prepared PS samples and (b) 58 day aged samples as a function of etching current density with detection wavelength of $520 \mathrm{~nm}$. For each spectrum, the lifetime of the multiexponential decay curve is shown together with the relative portion of the fast and slow decay components. The profiles are offset purpose of comparison.

to the limitation of our apparatus, the lifetime smaller than about $10 \mathrm{~ns}$ is difficult to detect so that we cannot rule out the possibility that the very fast decay component, typically several picoseconds to several nanoseconds, may exist.

The PL decay profiles of 58 day aged samples at the same detection wavelength are shown in Fig. 4(b). For all the profiles, the fast decay component increases drastically compared with the as-prepared sample. It is notable that the profile of green PS becomes very similar to that of blue PS. The fact that the decay spectra for the two aged samples are nearly the same also reveals conclusively that there is a universal radiating center for these samples. It may be related to an oxygen-related defect. However, the exact configuration and structure of the defect that is responsible for blue emission is not clear for now. In Fig. 4, the decay profiles of the aged green and blue PS consist of two lifetime components, but in this case the slow decay component is negligible. This fact indicates that the decay profile of the two aged samples can be monoexponential. Indeed, we checked out the decay profile at a detection wavelength of $430 \mathrm{~nm}$ and found that monoexponential decay can describe the decay completely as shown in Fig. 5. The lifetimes are $20 \mathrm{~ns}$ for aged green PS and $24 \mathrm{~ns}$ for aged blue PS, respectively.

In our study, the as-prepared green PS shows a $\sim 0.1 \mu \mathrm{s}$ lifetime and the aged green PS shows a $\sim 20$ ns lifetime. The 


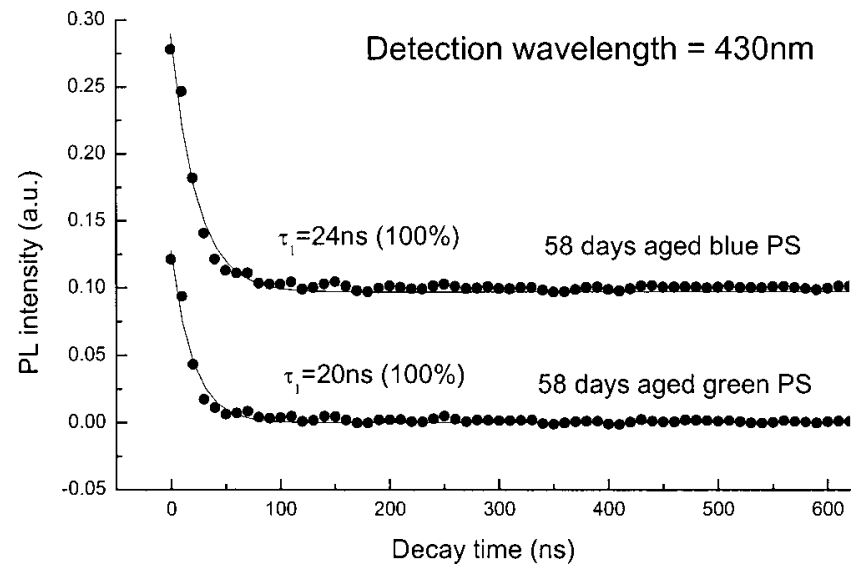

FIG. 5. Decay profiles of the aged green and blue PS at a detection wavelength of $430 \mathrm{~nm}$. Complete monoexponential decay behavior is clearly shown.

observed monoexponential decay profile at the detection wavelength of $430 \mathrm{~nm}$ suggests the possibility that the lifetime is almost the same for nanoparticle and oxygen-related defect.

\section{SUMMARY}

In summary, the aging behavior of the red, green, and blue-emitting PS prepared by Zn-aided electrochemical etching has been studied over a 3 month time span. The PL spectra and decay curves of aged samples show that there are two luminescent centers for $\mathrm{Zn}$-aided PS. One is the quan- tum confinement effect, which is responsible for the initial red and green emission and the other is the oxygen-related defect, which is responsible for the initial blue and aged green and blue emission. For the aged green and blue PS, the monoexponential decay profile is observed. The profile may result from two radiating states of a similar lifetime, which can be attributed to the quantum confined nanostructure and oxygen-related defect.

${ }^{1}$ T. Canham, Appl. Phys. Lett. 57, 1046 (1990).

${ }^{2}$ J. L. Gole, F. P. Dudel, D. Grantier, and D. A. Dixon, Phys. Rev. B 56, 2137 (1997).

${ }^{3}$ S. M. Prokes and W. E. Carlos, J. Appl. Phys. 78, 2671 (1995).

${ }^{4}$ Y. Fukuda, K. Furuya, N. Ishikawa, and T. Saito, J. Appl. Phys. 82, 5718 (1997).

${ }^{5}$ B. Zou, J. Wang, C. Liu, J. Z. Zhang, and M. A. El-Sayed, Phys. Rev. B 62, 16595 (2000).

${ }^{6}$ Y. H. Xie, W. L. Wilson, F. M. Ross, J. A. Mucha, E. A. Fitzgerald, J. M. Macauley, and T. D. Harris, J. Appl. Phys. 71, 2403 (1992).

${ }^{7}$ J. Linnros, N. Lalic, A. Galeckas, and V. Grivickas, J. Appl. Phys. 86, 6128 (1999)

${ }^{8}$ Y. Fukuda, K. Furuya, N. Ishikawa, and T. Saito, J. Appl. Phys. 82, 5718 (1997)

${ }^{9}$ L. Tsybeskov, Ju. V. Vandyshev, and P. M. Fauchet, Phys. Rev. B 49, 7821 (1994).

${ }^{10}$ S. Komuro, T. Kato, T. Morikawa, P. O'Keeffe, and Y. Aoyagi, Appl. Phys. Lett. 68, 949 (1996).

${ }^{11}$ J. H. Stathis and M. A. Kastner, Phys. Rev. B 35, 2972 (1987).

${ }^{12}$ K. Y. Suh, Y. S. Kim, S. Y. Park, and H. H. Lee, J. Electrochem. Soc. 148, C439 (2001).

${ }^{13}$ H. I. Liu, D. K. Biegelsen, F. A. Ponce, N. M. Johnson, and F. A. Pease, Appl. Phys. Lett. 64, 1383 (1994).

${ }^{14}$ P. Li, G. Wang, Y. Ma, and R. Fang, Phys. Rev. B 58, 4057 (1998).

${ }^{15}$ Y. S. Kim, K. Y. Suh, H. Yoon, and H. H. Lee (submitted for publication). 